... Pierre-Yves Maillard, conseiller d'Etat et directeur de la santé du canton de Vaud, président de la Conférence suisse des directrices et directeurs cantonaux de la santé (CDS)

\title{
«La liberté thérapeutique est très importante»
}

Daniel Lüthi

Texte et photos

danielluethi@gmx.ch
Les politiciens engagés aiment à parler de politique, et Pierre-Yves Maillard, de politique de la santé. Mais tentons malgré tout de commencer l'entretien par des questions plus personnelles. Son origine sociale par exemple, qui pourrait bien avoir joué un rôle déterminant dans cette remarquable carrière politique. Le politicien ne le nie pas; et, confortablement installé derrière un imposant bureau, il évoque son enfance avec enthousiasme: «Mon grand-père paternel était maçon, et mon grand-père maternel agriculteur.
Comme je n'étais pas très habile de mes mains, ma mère m'envoyait chaque fois passer les vacances dans la ferme de ses parents.» Là-bas, le futur conseiller $\mathrm{d}^{\prime}$ Etat n'a pas seulement appris à conduire un tracteur, il a apparemment aussi appris à travailler. C'est auprès des ouvriers - et donc aussi auprès de son père qui a d'abord travaillé dans un garage, puis dans une école en tant que concierge - que se sont cristallisées les valeurs fondamentales de sa future activité politique: «Oui, mes racines sociales sont le fondement

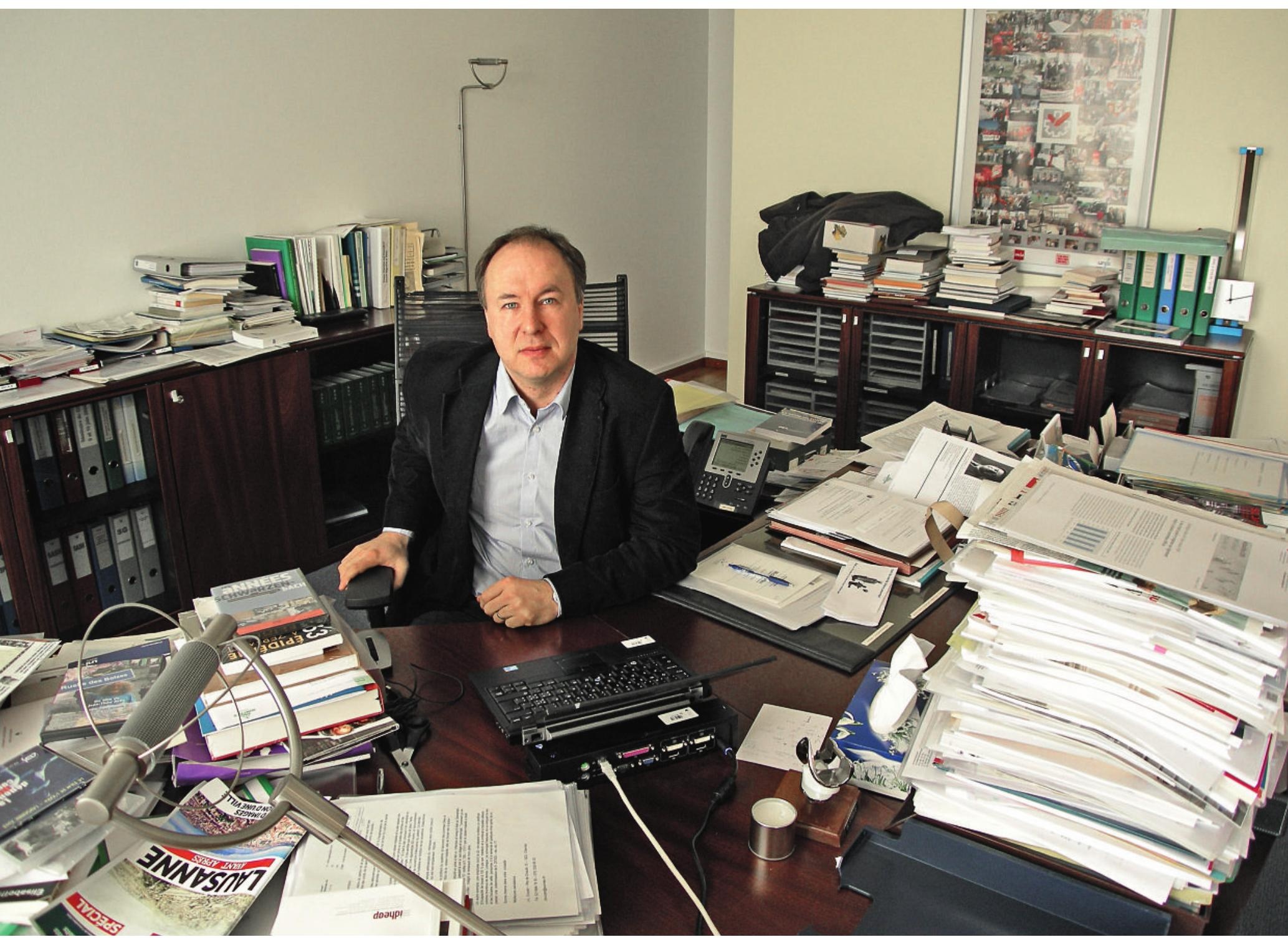


de mon engagement.» Et voilà que nous touchons déjà au sujet de prédilection de ce politicien engagé.

\section{Le monopole est une chance}

«L'idée du «service public équilibre les lois de l'économie de marché», déclare P.-Y. Maillard. Nous comprenons alors que ces propos pourraient rapidement être suivis par un plus long discours politique, comme le quadragénaire a l'habitude d'en tenir. Que veut-il dire, concrètement? «Prenons la Poste par exemple, qui couvre des besoins de base de la population. Elle est importante même dans les endroits où elle n'est pas rentable.» Ou le marché de l'électricité, que le peuple suisse a refusé de libéraliser lors d'une votation en 2002; «un succès socialiste», selon P.-Y. Maillard. Ou encore les CFF: «Nous avons probablement les meilleurs chemins de fer au monde», écrit-il avec enthousiasme dans son livre consacré aux faiblesses de l'assurance-maladie [1].

\section{«Oui, mes racines sociales sont le \\ fondement de mon engagement.»}

Dans certains domaines existentiels, l'idée centrale est la même: les monopoles contrôlés démocratiquement peuvent favoriser l'économie sociale alors que l'économie de marché et le néo-libéralisme le plus souvent purs lui nuisent. Transposés à la politique de la santé de ce pays, cela signifie que «le rôle de l'Etat en matière de planification et de régulation doit être renforcé. Car dans ce marché faussé, sous perfusion de financements publics, une offre dérégulée ferait exploser les coûts et les inégalités.» Pour les caisses-maladie, en éternelle concurrence, cela signifie selon P.-Y. Maillard que les principes de solidarité sont remplacés par des principes de rentabilité, et que la sélection des risques, basée sur le profit, engendre une médecine à deux vitesses, contraire aux fondements sociaux. C'est pourquoi il défend activement le projet de caisse unique, qui n'a jusqu'à présent obtenu ni la majorité du Parlement ni celle du peuple.

Mais P.-Y. Maillard est un battant, plus motivé que freiné par les divergences d'opinion. Un hebdomadaire romand l'a d'ailleurs qualifié de «bulldozer». Philosophe, il déclare: «Je ne suis pas une girouette qui change constamment de direction. Cela m'a valu déjà à l'âge de 30 ans d'être traité par certains d'‘archaïque`...»

\section{«Le système comporte des erreurs»}

Mais retournons à la politique suisse: «Le système actuel comporte de graves erreurs constitutives», avance P.-Y. Maillard. Lorsque nous lui demandons un exemple, il nous confie une anecdote tirée de son passé de secrétaire régional du syndicat FTMH pour

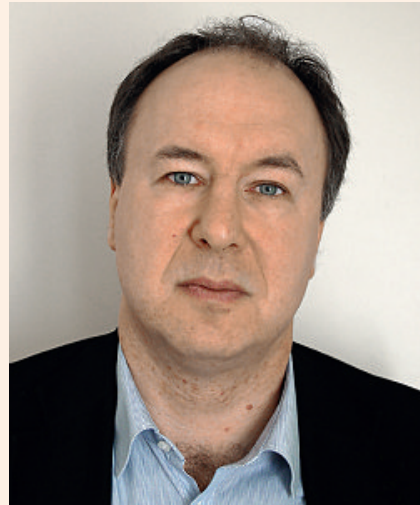

\section{Pierre-Yves Maillard.}

Pierre-Yves Maillard est né en 1968 à Lausanne.

Après des études et une formation de maître secondaire, il enseigne le français, I'histoire et la géographie. II entre très tôt en politique: il adhère au parti socialiste à 20 ans, devient membre du Conseil communal de Lausanne à

22 ans. Il est ensuite conseiller national de 1999

à 2004 et vice-président du PS de 2004 à 2008 .

En 2004, P.-Y. Maillard est élu au Conseil d'Etat vaudois remportant les élections face au représentant de I'UDC, Martin Chevallaz. Il est depuis, au sein du collège de sept conseillers d'Etat, chef du Département de la santé et de l'action sociale.

Pierre-Yves Maillard est marié et père de deux enfants (âgés de deux ans et de trois ans et demi). II vit avec sa famille à Renens, près de Lausanne.

les cantons de Fribourg et de Vaud, de 2000 à 2004: «Nous exploitions alors une petite caisse-maladie. Elle avait un taux de couverture de $100 \%$ et les primes les plus basses de la région. Une excellente situation donc, pour toutes les parties. Nous avons doublé le nombre d'adhérents, les réserves ont alors diminué de moitié, mais cela restait confortable. Puis, nous avons demandé une augmentation de primes de $5 \%$ à l'office fédéral compétent. Telle a été sa réponse: les autres caisses vont toutes augmenter leurs primes de $10 \%$; vous ne pouvez plus être si bon marché. Par ailleurs, $10 \%$ n'aurait pas suffi à notre caisse, parce que nous 
aurions encore été trop peu chers - nous avons donc dû demander $17 \%$ de plus. Incroyable, non? Voilà pourquoi de nombreuses petites caisses ont disparu.»

Du reste, P.-Y. Maillard a failli faire une formation dans le domaine de la santé: «J'ai envisagé d'étudier la médecine», se souvient-il. Il a ensuite changé d'avis, en raison de la durée des études: «Je ne voulais pas être à la charge de mes parents et préférais être financièrement indépendant aussi vite que possible».

\section{«Le système actuel comporte de graves erreurs constitutives.»}

\section{Economie de marché chez les médecins?}

Et aujourd'hui, que lui vient-il à l'esprit lorsqu'il pense à la médecine et aux médecins? «Un métier absolument fascinant et important», s'extasie-t-il, mais à nouveau, il en perçoit aussi rapidement les dangers: «Aujourd'hui, les médecins suisses disposent encore des bases leur permettant de mettre le bien-être de leurs patientes et de leurs patients au premier plan. Avec les nouveaux modèles, les considérations commerciales pourraient prendre le dessus.»

Plus concrètement: «Dans une situation où $10 \%$ des patients génèrent $70 \%$ des coûts, où les caissesmaladie sont en concurrence et veulent, en d'autres termes, éviter autant que possible les mauvais risques, le managed care géré par les caisses est un système dangereux, car il peut enrôler les soignants dans la stratégie de sélection des risques.» Là encore, il rejette clairement le libéralisme économique dans un domaine qui touche aux besoins de base de la population, d'autant plus surprenant qu'il prône une vision plutôt libérale de la profession de médecin lorsqu'il réitère que «la liberté thérapeutique est très importante».

Mais en même temps, P.-Y. Maillard insiste sur le fait que l'accès aux principales prestations médicales doit être garanti à toutes et à tous: «Si je ne peux pas me payer de Ferrari à 150000 francs, je peux vivre sans. Mais si j'ai besoin de la même somme pour un traitement contre le cancer, je dois pouvoir l'obtenir.» Et ce, indépendamment de la situation financière personnelle dans laquelle on se trouve: «Il n'est tout simplement pas normal qu'une personne débourse $2 \%$ de ses revenus pour sa prime d'assurance-maladie, alors qu'une autre en débourse $20 \%$ pour les mêmes prestations.»

P.-Y. Maillard - en digne représentant d'un gouvernement cantonal - souhaite que la régulation du système de santé soit autant que possible entre les mains des cantons, à la fois dans les domaines hospitalier stationnaire et ambulatoire hospitalier. Dans le canton de Vaud, cela a fait ses preuves: «En 2003, les coûts étaient encore supérieurs de $20 \%$ à la moyenne suisse; à présent, ils ne la dépassent plus que de $10 \%$. Les coûts générés par les services de soins à domicile, les hôpitaux stationnaires et les EMS étaient alors 8\% au-dessus de la moyenne; à présent, ils se situent $5 \%$ en-dessous.»

Un autre chiffre laisse par contre P.-Y. Maillard perplexe: «Pourquoi les vaudois consomment-ils environ $20 \%$ de médicaments de plus que les zurichois par exemple? Et ce, alors que les médecins ne remettent pas de médicaments dans leurs cabinets et que les vaudois se rendent un peu moins souvent chez le médecin que les suisses allemands?» Il a mandaté une étude à ce sujet pour en avoir le cœur net.

\section{«Le rôle de l'Etat en matière de planification et de régulation doit être renforcé.»}

\section{La santé du directeur de la santé}

Rapprochons-nous à nouveau de l'homme plutôt que du politicien. Le premier semble faire la moue, nous nous adressons donc au directeur de la santé: «Et vous, que faites-vous pour 〈réguler〉 votre santé?» «J'ai eu la chance de pouvoir entrer en politique jeune et donc en pleine forme. A présent, c'est plus fatigant. Avec deux enfants en bas âge, la vie de famille n'est pas toujours reposante. Et il m'arrive certainement de dépenser plus d'énergie que je ne le devrais. Mais je suis encore jeune.» Autrement dit: se ressourcer n'est pas une priorité. Il ne joue par exemple plus au jass. Mais il écoute parfois encore de la musique: «Mon musicien préféré est Bruce Springsteen.»

Et quels sont les projets de cet homme qui a tant accompli sur le plan professionnel dans ses jeunes années? A entendre sa réponse, nous réalisons que cette question ne le préoccupe pas pour le moment. Il hausse les épaules et déclare simplement: «On verra.»

\section{La prochaine «Rencontre avec...»}

A la fin de chaque mois, le Bulletin des médecins suisses présente une personnalité qui s'implique dans la santé publique. En avril, Daniel Lüthi parlera de sa rencontre avec Dominic Huser: médecin-assistant en chirurgie à I'hôpital cantonal de Lucerne à Sursee, Dominic Huser est probablement le plus jeune médecin en Suisse. 